\title{
DISCUSSION ON MULTI RESOLUTION WAVELET SMOOTHING OF FREE CURVE
}

\author{
Chen Yong ${ }^{1}$, Zhang $\mathrm{Xu}^{2}$ \\ ${ }^{1}$ College of Mechanical Engineering, Shanghai University Of Engineering Science, Shanghai, China \\ ${ }^{2}$ College of Mechanical Engineering, Shanghai University Of Engineering Science, Shanghai, China
}

\begin{abstract}
Fairing is one of the design requirements of industrial products. The Fairing of curves and surfaces has important influence on product quality and physical properties. In recent years, wavelet based curve and surface fairing methods have received extensive attention in academic circles. Wavelet analysis is a widely used mathematical analysis tool based on the theory of signal analysis and processing. This method mainly utilizes the multi-resolution characteristic of wavelet, and the wavelet smoothing of curves and surfaces can be regarded as filtering the curves, removing the high frequency and retaining the low frequency. So as to achieve the smoothing effect of curves and surfaces. In this paper, we mainly discuss the multiresolution wavelet smoothing problem of free curve.
\end{abstract}

Keywords: - Multiresolution analysis, wavelet smoothing, B-spline

\section{INTRODUCTION}

With the rapid development of reverse engineering technology, surface reconstruction is becoming more and more important. The accuracy is becoming more and more demanding. However, due to the limitation of measurement technology, the process of prototype digitization will take place. Inevitably, all kinds of errors are generated, thus affecting the reconstructed surfaces. Fairing. Curve and surface smoothing operation is a direct and effective way to improve the quality of reconstruction.

Multiresolution analysis is a kind of internal correlation that can depict data.The new time frequency analysis mathematical tool has good time domain locality and frequency domain locality. With the maturity of the technology, multiresolution analysis has been applied to computer graphics. In 1994, Quak applied multi-resolution analysis tools to solve the problem of curve decomposition and reconstruction based on closed-interval B-spline wavelet for $2^{\mathrm{j}}+3$ control vertices. ${ }^{[1]}$ Aiming at the new field of multiresolution fairing, many scholars both here and abroad have studied it. Based on the binary wavelet smoothing algorithm, Ceruti ${ }^{[2]}$ constructs a fast multi-levelof-detail(Levels of detail, LOD) filter bank and realizes the wavelet smoothing of class-A curves. Based on the classical multi-resolution analysis theory and combined with the second generation lifting lattice wavelet, Pan Yangyu ${ }^{[3]}$ deduced the lifting lattice wavelet representation method of quasi-uniform cubic B-spline curve by dividing, predicting and updating the lifting steps, and realized the fairing of quasi-uniform cubic B-spline curve. Zhao Gang ${ }^{[4]}$ and others applied non-uniform B-spline wavelet transform to the automatic smoothing of non-uniform B-spline curves. By decomposing the details of the curves obtained by wavelet transform again and setting the threshold automatically, the wavelet details can be repaired under certain constraints. Then we reconstruct the wavelet details and get the smooth curve. Then we use the control points of the smooth curve as the input control points to calculate iteratively until a better fairing effect appears. The algorithm smoothes general nonuniform B-spline curves automatically by iteration. It is an automatic Fairing Algorithm Based on non-uniform B spline wavelets.

The precision and smoothness of curves are conflicting, so it is necessary to sacrifice the quality of curves to achieve higher requirements on the other hand. ZengZhuo ${ }^{[5]}$ proposed a curve fitting method based on cubic B-spline wavelet. The data information of curve control point coordinates was processed by wavelet, and the highfrequency detail information was removed. The lowfrequency subject information was obtained. The data features of the new control points with about half the original control points were used. This not only guarantees the fitting accuracy, but also guarantees the fitting smoothness, which is very helpful to improve the accuracy of NC machining. It is a method of fitting accuracy, smoothness and machining accuracy.

\section{BASIC KNOWLEDGE OF B-SPLINE WAVELET TRANSFORM}

Wavelet analysis is developed on the basis of Fourier analysis. The wavelet analysis of modern meaning began in the late 1970s.In recent years, a new method of curve and surface modeling based on wavelet analysis has emerged. Lyche $^{[6]}$ studies the orthogonal decomposition of B spline space on bounded closed interval. Lyche constructs nonuniform B-spline wavelets with minimum support intervals, thus increasing the flexibility of wavelet bases. 


\subsection{Multiresolution Analysis}

$\left\{V_{j}\right\}$ The sequence of finite dimensional subspaces of $L^{2}[a, b]$. if

$$
\begin{aligned}
& V_{0} \subset V_{1} \subset V_{2} \subset \ldots \ldots ; \\
& \bigcup_{j=0}^{\infty} V_{\mathrm{j}}=L^{2}[a, b] \\
& \mathrm{V}_{\mathrm{j}} \text { was based on Riesz basis }\left\{\varphi_{\mathrm{i} . \mathrm{j}}\right\}^{\mathrm{nj}}{ }_{\mathrm{i}=1}
\end{aligned}
$$

For arbitrary $\mathrm{C}_{\mathrm{j}}=\left(\mathrm{C}_{1 . \mathrm{j}} \ldots \ldots \mathrm{C}_{\mathrm{nj}, \mathrm{j}}\right)^{\mathrm{T}}, \mathrm{K}_{1}\|C j\|_{2 \leq} \mathrm{C}_{\mathrm{i} . \mathrm{j}}$

$$
\mathrm{K}_{1}\|C j\|_{2} \leq\left\|\quad \sum_{i=1}^{n j} C_{i . j} \varphi_{i . j} \quad\right\|_{2} \leq \mathrm{K}_{2}\|C j\|_{2}
$$

The subspace sequence $\left\{\mathrm{V}_{\mathrm{j}}\right\}$ is multiresolution analysis of $L^{2}[a, b]$. The basis function $\left\{\varphi_{\mathrm{i} . \mathrm{j}}\right\}_{\mathrm{i}=1}^{\mathrm{nj}}$ is usually called scaling function.

\subsection{Nonuniform B-spline Wavelet Transform}

Due to the strict restriction of the number of control vertices in Fairing Algorithm Based on quasi-uniform B-spline, its practical application in curve and surface modeling is limited. Its generality is not as good as that based on nonuniform B-spline wavelet. To solve this problem, a smoothing algorithm based on nonuniform B spline wavelets is introduced in this paper.

Definition of $\mathrm{k}$ degree non-uniform B spline curve

$$
r_{j}(u)=\sum_{i=0}^{n} C_{i}^{j} \Phi_{i}^{j}(u)
$$

The B-spline basis function $\Phi_{i}^{j}(u)(\mathrm{i}=0,1,2, \ldots, \mathrm{n})$ is defined by node vector $\left[\mathrm{u}_{0}, \mathrm{u}_{1}, \mathrm{u}_{2}, \ldots, \mathrm{u}_{\mathrm{n}+\mathrm{k}+1}\right]$. Make a hypothesis: $\Phi^{j}=\left[\Phi_{0}^{j}(u), \Phi_{1}^{j}(u) \ldots \ldots \Phi_{n}^{j}(u)\right], \mathrm{C}^{\mathrm{j}}=\left[\mathrm{C}_{0}^{\mathrm{j}}, \mathrm{C}_{1}{ }^{\mathrm{j}}\right.$, $\left.\mathrm{C}_{2}{ }^{\mathrm{j}} \ldots \ldots \mathrm{C}_{\mathrm{n}}{ }^{\mathrm{j}}\right]$; So the $r_{j}(u)$ can also be expressed as

$$
r_{j}(u)=\Phi^{\mathrm{j}} \mathrm{C}^{\mathrm{j}}
$$

Let $V^{j}$ represent the space formed by the nonuniform Bspline basis function $\Phi^{j}$, and the dimension of $\mathrm{V}$ is $\mathrm{n}+1$.

Nonuniform B-spline wavelet transform requires 3 steps ${ }^{[7]}$. Choose scaling function $\Phi^{j}$, that is, the basis function of space $\mathrm{V}$.

The inner product of any two functions $\mathrm{f}$ and $\mathrm{g}$ in space $V^{j}$ is defined and the wavelet space $W^{j-1}$ of orthogonal complement of space $\mathrm{V}^{\mathrm{j}-1}$ is defined by the inner product. Select the basis function of wavelet space $W^{j-1}$, that is, wavelet function $\Psi^{j^{-1}}$.

Since space $V^{j-1}$ is a subspace of $V^{j}$, the basis function $\Phi^{j-1}$ in space $V^{j-1}$ can be expressed linearly by the basis function_of space $V^{j}$. That is, $\Phi^{j-1}=\Phi^{j} P^{j}$, where $P^{j}$ can be obtained by node insertion algorithm.
Definition inner product:

$$
\langle f . g\rangle=\int_{0}^{1} f(u) g(u) d u \quad f, g \in V^{j}
$$

Where $V^{j-1}$ has unique orthogonal complement space in $V^{j}$, it is called $W^{j-1}$. That is, $V^{j}=V^{j-1} \oplus W^{j-1}, W^{j-1}$ is called wavelet space. $W^{j-1}$ is the subspace of space $V^{j}$, whose base function $\Psi^{j^{-1}}$ can be linearly expressed by the basis function $\Phi^{j}$ of space $V^{j}$. That is $\Psi^{j-1}=\Phi^{j} Q^{j}$.

The nonuniform B-spline curve $\gamma_{\mathrm{j}}(\mathrm{u})$ can be expressed as

$$
\gamma_{\mathrm{j}}(\mathrm{u})=\Phi^{j} C^{j}=\Phi^{j-1} C^{j-1}+\Psi^{j-1} D^{j-1}(2-5)
$$

Where $\Phi^{j-1} C^{j-1}$ is called the scale part of a curve, $\Psi^{j-1} D^{j-1}$ is called The detail part of the curve. Because $\Phi^{j-1}=\Phi^{j} P^{j}, \Psi^{j-}$ ${ }^{l}=\Phi^{j} Q^{j}$, we know $C^{j}=P^{j} C^{j-1}+Q^{j} D^{j-1}, P^{j} C^{j-1}$ is the corresponding scale coefficient matrix, and $Q^{j} D^{j-1}$ is the coefficient matrix of detail.

\section{ALGORITHM STEPS}

The automatic smoothing algorithm based on non-uniform B-spline wavelets is as follows:

Step 1: Choose wavelet space, decompose original curve $\gamma_{\mathrm{j}}(\mathrm{u})$ into scale part

$\gamma_{\mathrm{j}-1}(\mathrm{u})$ and detail $\operatorname{part} \beta_{\mathrm{j}-1}(\mathrm{u})$ by non-uniform B-spline wavelet. For non-uniform B splines, we need to select a subsequence of the original node vector.

Step 2: The detail $\operatorname{part} \beta_{\mathrm{j}-1}(\mathrm{u})$ is decomposed into wavelet scale $\operatorname{part} \beta_{\mathrm{j}-2}(\mathrm{u})$ and wavelet detail $\operatorname{part} \beta_{\mathrm{j}-2}^{\beta}(\mathrm{u})$ by lower order non-uniform B-spline wavelet.

Step 3: Repairing wavelet details. The wavelet detail part $\beta^{\beta}{ }_{\mathrm{j}-}$ ${ }_{2}(\mathrm{u})$ is made into a set of signals and filtered again. Integral restoration method: The threshold and compression coefficients are set automatically by the wavelet detail coefficient matrix, and $\mathrm{M}$ is made the coefficient matrix of the wavelet detail part, and then filtered. In general, the larger the modulusof $M(i, j)$, the less smooth the curve at its corresponding node.Local smoothness can be achieved by compressing $M(i, j)$.

Step 4: Reconstructing the curve. Reconstructing the modified wavelet details.

Step 5: Iterative fairing curve. The new control vertex obtained by smoothing is used as input control vertex for iterative smoothing again until the iterative termination condition is satisfied. The termination condition is generally achieved by setting error limit error and the maximum number of iterations $\mathrm{N}$ ( $\mathrm{N}$ is generally set to 10). When the iteration error is greater than error or the number of iterations is greater than $\mathrm{N}$, the algorithm terminates automatically. Since each smoothing can achieve better smoothing effect than the previous one, it can achieve better smoothing effect than other algorithms through multiple iterations. 
The curve can be decomposed into two parts: scale and detail, which greatly affect the smoothness of the curve. The greater the modulus of the corresponding detail part of a node, the less likely the curve will be at this node. But if we adjust the detail coefficient of this node directly, sometimes the smoothing effect is not ideal. The reason is that the Bspline basis function has a certain support interval, and the fairness of the curve at this node is mainly determined by the detail coefficients corresponding to the nodes and the surrounding nodes. In view of this, this paper decomposes the detail part again, gets the wavelet scale part and the wavelet detail part after decomposition, then reduces the detail part of the curve by repairing the wavelet detail part, thus achieving the goal of smoothing the curve. The smooth curve is smoothed again until the better smoothing effect is achieved. More smooth information can be filtered out by successive smoothing.

The new control vertex obtained by smoothing is used as input control vertex for iterative smoothing again until the iterative termination condition is satisfied. The termination condition is generally achieved by setting the error limit error and the maximum number of iterations $\mathrm{N}(\mathrm{N}$ is generally set to 10). When the iteration error is greater than error or the iteration number is greater than $\mathrm{N}$, the algorithm automatically terminates.

Figure 1 shows an example of smoothing 3 iterations of a curve using this algorithm. Figure 1 a shows 3 non-uniform B spline curves with 24 control points. Figure $1 \mathrm{~b}$ shows the result curve obtained from the 3 iteration smoothing algorithm. As shown in Figure $1 \mathrm{c}$ and d, the smoothness of curves is improved by first fairing. From Fig. 1 e f, the second smoothness is much better than the first smoothness, and the third smoothness achieves a good result.

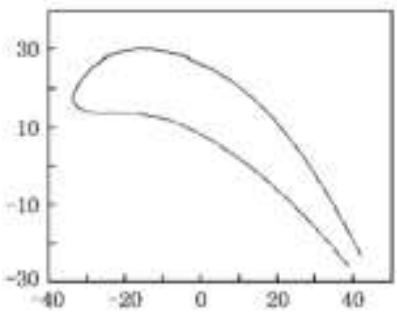

a) Original curve

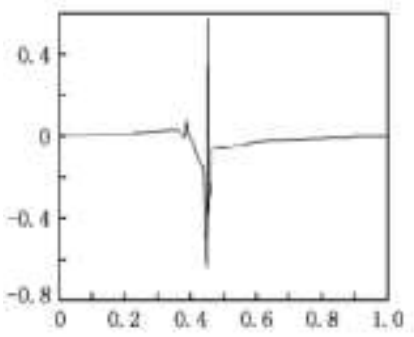

c) Original curvature graph

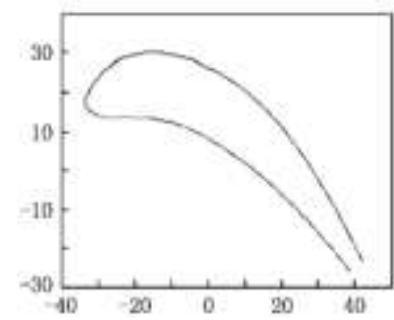

b) Fairing curve

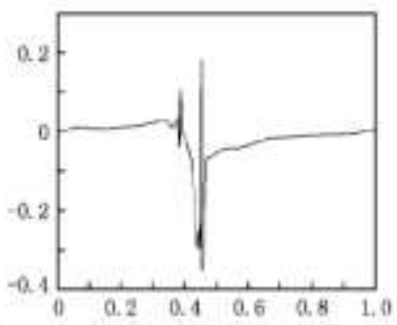

d) Primary fairing curvature graph

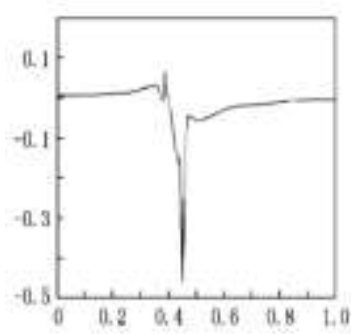

e) Two fairing curvature diagram

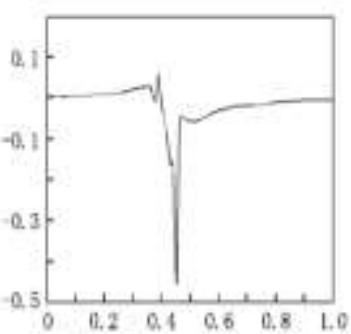

f) Two fairing curvature diagram
Fig 1: Curvature smoothing effect analysis diagram

\section{MULTIRESOLUTION WAVELET FAIRING PRECISION CONTROL}

In this paper, we can directly estimate the error of the curve according to the change of the control points before and after the fairing.

$$
\begin{gathered}
\max \left|\gamma_{j}(u)-\bar{\gamma}_{j}(u)\right|=\max \left|\Phi^{j}\left(C^{j}-\bar{C}^{j}\right) \quad\right|= \\
\max \left|\sum_{i=0}^{n} d^{i} \Phi_{i}^{j}(u)\right|
\end{gathered}
$$

The $\left(C^{j}-\bar{C}^{j}\right)=\left[d_{0}, d_{1} \ldots \ldots d_{n}\right.$

If:

$$
m=\max _{i}\left|\Phi^{j}\left(C^{j}-\bar{C}^{j}\right) \quad\right| 0 \leq \mathrm{i} \leq \mathrm{n}
$$

Then:

$\max \left|\quad \gamma_{j}(u)-\bar{\gamma}_{j}(u)\right| \leq m \times \max \left|\quad \sum_{i=0}^{n} \Phi_{i}^{j}(u)\right| \leq \mathrm{m}=$ $m=\max _{i}\left|\left(C^{j}-\bar{C}^{j}\right)\right|(4-2)$

For:

$$
\left|\sum_{i=0}^{n} \Phi_{i}^{j}(u)\right|=1
$$

And:

$$
\Phi_{i}^{j}(u) \geq 0
$$

That is, the maximum error before and after curve smoothing is less than or equal to the maximum movement of the control point. More precisely, the error of a curve at a point is less than or equal to the maximum displacement of $k+1$ control vertices defining the curve segment. When the two curves are very close, the global error between the two curves can be approximated by

$$
\text { error }=\sum_{i=1}^{n} \mid\left(C^{j}-\bar{C}^{j}\right)
$$




\section{CONCLUSION}

The algorithm in this paper achieves the automatic smoothing effect by setting the threshold automatically. With the increase of iteration times, the smoothing effect becomes better and better. The automatic smoothing effect of the curve is achieved. The algorithm in this paper can achieve better fairing effect by multiple iterations, and can adjust the number of iterations automatically through the fairing termination condition more flexibly to achieve automatic fairing of curves.

\section{REFERENCES}

[1] Quak E, Weyrich N Decomposion and reconstruction algorithms for splines wavelets on a bounded interval [J] Applied and Computational Harmonic Analysis, 1994.1(3):217*-231

[2] Ceruti A, Liverani A, Caligiana G. Fairing with neighbor-hood LOD filtering to upgrade interactively $\mathrm{B}$-spline into class-A cure [J]. International Journal on Interactive Design and Manufacturinhg,2012(11):1-9

[3] Pan Yangyu, Research on the method of B-spline curves fairness based on lifting scheme[J]. Machiney,2010,37(5)6-8.(in Chinese)

[4] Zhao Gang, Wavelet-Based Automatic Fairing Algorithm For Non-uniform B-spline Curve[J]. Journalof Computer-Aided Design and ComputerGraphics, 2010,22(11)1925-1931.(in Chinese)

[5] ZengZhuo, A Research on Curve Fitting of Cubic Bspline Wavelets [J].Mechanical Science and Technology for Aerospace Engineering, 2018, 37(6)891-895.(in Chinese)

[6] LycheT, M orken K , Quak E .Theory and algorithms fornon-uniform spline wavelets[ C] Proceeding $\mathrm{s}$ of MultivariateApproximation and Applications. Cam bridge: CambridgeUniversity Press, $2001: 152-187$

[7] Stollnitz E J, Derose T D, Salesin D H. Wavelets forcomputer graphics-theory and applications [ D] . SanFrancisco:Morgan Kaufmann , 1996 